\title{
Gait Recognition from Front and Back View Sequences Captured Using Kinect
}

\author{
Pratik Chattopadhyay $^{1}$, Shamik Sural ${ }^{1}$, and Jayanta Mukherjee ${ }^{2}$ \\ 1 School of Information Technology, IIT Kharagpur, India \\ 2 Dept. of Computer Science \& Engineering, IIT Kharagpur, India \\ \{pratikc, shamik@sit.iitkgp.ernet.in, \\ jay@cse.iitkgp.ernet.in
}

\begin{abstract}
In this paper, we propose a key pose based gait recognition approach using skeleton joint information derived from the depth data of Kinect. We consider situations where such depth cameras are mounted on top of entry and exit points, respectively capturing back and front views of subjects who enter a zone under surveillance. Three dimensional geometric transformations are used to map the skeleton images captured from the back view to an equivalent front view. A gait cycle is divided into a number of key poses and the trajectory followed by each skeleton joint within a key pose is used to derive the gait features for that particular pose. For recognizing a subject, available key poses are compared with the corresponding key poses of the training subjects. The proposed method has higher accuracy than other competing approaches.
\end{abstract}

Keywords: Gait Recognition, Kinect, Geometric Transformation, Skeleton Joint, Key Pose.

\section{Introduction and Background}

Gait recognition is the process of identifying an individual from his style of walking. Suitability of gait as a biometric feature in security applications is established by the fact that unlike other biometric methods like face detection, iris scan and finger print detection, close interaction with a subject is not required in gait recognition. Gait can be identified from a distance and even with low resolution video sequences.

Most of the existing work on gait recognition considers capturing of video sequences in the fronto-parallel (side) view, since maximum gait information during walking is contained in this view [1, 2. It has, however, been pointed out that the assumption on the availability of gait features from the frontoparallel view is too restrictive especially in situations where gait is presumed to be the biometric of choice, i.e., in places like airports and railway stations [3]. In such places, people are often directed to enter into secured zones one at a time like walking through a metal detector gate. Since it is easier to control the flow of persons at the entrance/exit of a security zone, surveillance cameras mounted above the entry/exit point can record a better view of a subject entering/leaving 
the zone. Such type of camera positioning captures the frontal view (back/front view) of an individual.

Research work strictly based on frontal gait recognition includes the curve spread method [4, methods based on intra and inter frame rectangular size distribution [5], the largest Lyapunov exponents [6], three dimensional moments from silhouette shape variations [7] and a spherical space model for gait recognition [8]. These frontal gait recognition procedures have worked with gait videos captured by RGB video camera, and have shown accuracy to a certain extent. Frontal gait recognition based on silhouette shape and depth has started after RGB-D cameras like Kinect were made publicly available [9. Partial volumetric reconstruction of the depth data provided by Kinect has been used to construct Gait Energy Volumes (GEV) 3]. To preserve the dynamic information of gait at a higher resolution than what was considered in [3, Pose Depth Volume (PDV) 10] has been recently developed. These methods are computationally intensive as they operate at the voxel level of granularity. A comparatively faster gait recognition procedure based on the covariance of the skeleton joint trajectories provided by Kinect [1] has been proposed in [12].

The present study also makes use of the skeleton joint positions but the kinematic information of gait is preserved at a higher resolution by dividing a gait cycle into a number of key poses and constructing a gait feature corresponding to each such key pose.

The organization of the rest of the paper is as follows. Section 2 presents the proposed method for estimation of key poses, whereas construction of gait features and human recognition using the proposed feature are detailed in Section 3. The experimental setup together with results is presented in Section 4 Concluding remarks and future scope of work are pointed out in Section 5 .

\section{Key Pose Estimation}

Gait is a repetitive pattern followed during walking where each gait cycle covers two strides: the right foot forward and the left foot forward. Key poses are representatives of an entire gait cycle that characterize the gait cycle by maintaining sufficient variability between any two consecutive key poses. In this section, we describe the steps used in key pose estimation.

\subsection{Skeleton Alignment and Feature Vector Construction}

Let the angle of inclination of the skeleton with respect to the $Y$ axis of the Kinect coordinate system be $\alpha$. This inclination angle always causes the skeleton to be tilted at an angle with respect to the $Y Z$ plane of the Kinect coordinate system. But for frontal view gait videos, any inclination with respect to the $X Y$ plane of the coordinate system can be ignored. To make key pose estimation and gait recognition procedures invariant to camera positioning, transformation of the skeleton to a uniform viewpoint is accomplished using a three dimensional geometric transformation. In the present context, the segment joining the torso 
to neck of each skeleton is aligned along the $X Y$ plane of the Kinect coordinate system. Let the $3 \mathrm{D}$ coordinates of the torso and the neck of a certain skeleton as provided by Kinect be $T\left(X_{t}, Y_{t}, Z_{t}\right)$ and $N\left(X_{n}, Y_{n}, Z_{n}\right)$, respectively. First, each joint of the skeleton is translated by an amount $\left(-X_{t},-Y_{t},-Z_{t}\right)$, so that the torso of the skeleton maps to the origin. The translated coordinates of the torso and the neck are now given by $T^{\prime}(0,0,0)$ and $N^{\prime}\left(X_{n}^{\prime}, Y_{n}^{\prime}, Z_{n}^{\prime}\right)$ respectively. Next, the skeleton is rotated about $Z$ axis by an angle $\alpha$ so that the rotated coordinates of the neck maps to the $X Y$ plane of the coordinate system. For any transformation along the $Y Z$ plane, the value of the $X$ coordinate does not alter. Thus, if $N^{\prime \prime}\left(X_{n}^{\prime}, Y_{n}^{\prime \prime}, 0\right)$ denotes the new transformed coordinates of the neck, the numerical value of $\alpha$ is given by:

$$
\alpha=\cos ^{-1}\left(Y_{n}^{\prime \prime} / L\right),
$$

where, $L$ is the length of the segment joint $N^{\prime}$ with the origin.

For determining the key poses, consider a set $S=\left\{S_{1}, S_{2}, \ldots, S_{N}\right\}$ of $N$ skeletons comprising of walking stances of a sufficiently large number of persons. These skeletons are available either from the front view or from the back view. Consider that $C$ skeleton joints are tracked for each subject. For each skeleton, we compute the direction cosines of each of its joints with respect to the torso (i.e., origin). For any two vectors $\vec{A}$ and $\vec{B}$, the unit vector pointing from $\vec{A}$ to $\vec{B}$ denoted by $\widehat{A B}$ is given by:

$$
\widehat{A B}=\widehat{l i}+m \widehat{j}+n \widehat{k},
$$

where, $l, m$ and $n$ are termed as the direction cosines of the vector $\overrightarrow{A B}$. Let $l_{c}, m_{c}$ and $n_{c}$ denote the direction cosines of the $c^{t h}$ joint with respect to the torso, where, $c=1,2,3, \ldots, C$. Let $V_{i}$ denote the feature vector obtained after concatenating the direction cosines of all the $C-1$ joints with respect to the torso for the $i^{\text {th }}$ skeleton. Thus, $V_{i}$ is represented as:

$$
V_{i}=\left[\begin{array}{lllllllllll}
l_{c}^{1} & m_{c}^{1} & n_{c}^{1} & l_{c}^{2} & m_{c}^{2} & n_{c}^{2} & \ldots & l_{c}^{C-1} & m_{c}^{C-1} & n_{c}^{C-1}
\end{array}\right]^{T},
$$

for $i=1,2, \ldots, N$. Similar feature vectors computed for all the $N$ skeletons in the training set are concatenated to form the feature set for key pose estimation. Since the skeleton joints must be selected in a specific order, during feature construction, if the skeleton joints are chosen in clockwise order for front view skeletons, the ordering of the same sequence of joints for back view skeletons will be anticlockwise. Again, for each skeleton obtained from the back view, the signs of the $X$ and $Z$ components of the direction cosines will be reversed when the front view direction cosines have to be computed from the back view.

\subsection{Key Pose Estimation by Feature Vector Clustering}

Constrained $K$-Means clustering [13] is applied on the feature vectors constructed from the skeletons to determine the key poses. The centroids of the clusters obtained after clustering are the key poses, which we term as skeleton 
key poses. The constraint imposed on the cluster assignment step of the clustering process is that, reassignment of any feature vector in a subsequent step is only valid for the immediately preceding or succeeding cluster indices. The skeleton key poses are labeled such that key pose 1 starts with the mid stance and key pose $K$ ends with the pose just before the mid stance.

\section{Gait Feature Extraction and Human Recognition}

For deriving the gait features, the transformed joint positions on each skeleton rather than the direction cosines relative to the torso are considered. This is because the transformed joint position coordinates preserve information regarding local motion characteristics at a higher resolution than the direction cosines. Since the torso is always fixed at origin, the mean position of each of the remaining $C-1$ joints over all the skeletons belonging to a particular key pose is used to form the feature vector for that key pose.

\subsection{Feature Extraction}

For an input training gait sequence of a subject, we consider unconstrained data where the front view or the back view sequence could be available. Three dimensional transformations as explained in Section 2 are applied on each skeleton and equivalent front view representation is derived for each skeleton from the back view.

To find the mapping between the key pose numbers and the skeleton sequence, dynamic programming based Viterbi path decoding algorithm is employed. This step assigns to each skeleton of the sequence a unique key pose number. The assignment preserves a cyclic ordering of natural numbers 1 to $K$. For example, if the $i^{t h}$ skeleton is assigned a key pose $k$, then the $(i+1)^{t h}$ skeleton must be assigned to either key pose $k$ or key pose $1+(k \bmod K)$. Let the sequence of $p$ skeletons $P=\left\{S_{n}, S_{n+1}, S_{n+2}, \ldots, S_{n+p-1}\right\}$ (which is a subset of the input sequence $Q=\left\{S_{1}, S_{2}, S_{3}, \ldots, S_{Q}\right\}$ ) of a certain subject map to the key pose $k, k$ $=1,2,3, \ldots, K$. Further, let $J_{c, r}\left(X_{c}^{r}, Y_{c}^{r}, Z_{c}^{r}\right)$ be the transformed coordinates of the $c^{t h}$ joint of the $r^{t h}$ skeleton $\left(S_{r}\right)$, that is mapped to the key pose $k$, where, $r=n, n+1, n+2, \ldots, n+p$ and $c=1,2,3, \ldots, C-1$. The gait feature vector $F_{k}$ corresponding to the $k^{t h}$ skeleton key pose is hence obtained by averaging the $X, Y$ and $Z$ coordinates of each of the $C-1$ joints of the skeletons that are mapped to the key pose $k$. Mathematically,

$$
F_{k}=\left[\begin{array}{lllllllllllll}
X_{1 k} & Y_{1 k} & Z_{1 k} & X_{2 k} & Y_{2 k} & Z_{2 k} & X_{3 k} & Y_{3 k} & Z_{3 k} & \ldots & X_{(C-1) k} & Y_{(C-1) k} & Z_{(C-1) k}
\end{array}\right]^{T} .
$$

where,

$$
X_{c k}=\frac{1}{p} \sum_{r=n}^{n+p-1} X_{r k}, \quad Y_{c k}=\frac{1}{p} \sum_{r=n}^{n+p-1} Y_{r k}, \quad Z_{c k}=\frac{1}{p} \sum_{r=n}^{n+p-1} Z_{r k},
$$

Similar feature vectors are constructed for all the $K$ key poses and are concatenated to form the training gait feature set. Thus, the $k^{t h}$ column of the training gait feature vector represents the gait feature of the subject for the $k^{\text {th }}$ key pose. 
Now, consider an input test sequence which could be obtained from the back view only, from the front view only, or from each of the two views. The transformed skeleton sequences recorded from the front view and/ or the back view are taken into consideration separately. Viterbi path decoder is applied on each of these two sequences in order to obtain the skeleton key poses of each skeleton in the sequence.

Construction of the test gait feature vector for a particular key pose follows a similar procedure as that during training, but the computation considers those skeletons that are available either from the front or the back view or from both the views.

\subsection{Recognition Using Proposed Feature}

Whichever frames of the partial gait cycles are obtained from the two cameras, they are mapped to the appropriate skeleton key poses and searching within the training set is restricted to these skeleton key poses only.

Let the training gait features be available for $\mathcal{S}$ subjects. Let $F_{1}^{i}, F_{2}^{i}, F_{3}^{i}, \ldots$, $F_{K}^{i}$ be the $K$ feature vectors corresponding to the $K$ skeleton key poses of the $i^{t h}$ subject, where, $i=1,2,3, \ldots, \mathcal{S}$. Suppose $F_{j}^{i}=\left[F_{1 j}^{i} F_{2 j}^{i} F_{3 j}^{i} \ldots F_{\mathcal{N} j}^{i}\right]^{T}$, where $\mathcal{N}=3 \times(C-1)$ and $j$ ranges from 1 to $K$. Suppose for an input test subject, walking stances belonging to only $l$ out of the $K$ skeleton key poses are available. It may be noted that certain key poses could be missing even after combining front and back view sequences due to occlusion or noisy video capture. Let $k_{1}$, $k_{2}, k_{3}, \ldots, k_{l}$ be the available skeleton key poses for the test subject, where, $1 \leq k_{m} \leq K, \forall m=1,2, \ldots, l$. Also, let us suppose that $F_{k_{1}}^{\prime}, F_{k_{2}}^{\prime}, F_{k_{3}}^{\prime}, \ldots$, $F_{k_{l}}^{\prime}$ be the respective gait features for the available skeleton key poses of the test subject computed in a manner similar to that explained in Section 3. The gait feature vectors corresponding to these available skeleton key poses of the test subject are compared with the feature vectors corresponding to the same skeleton key poses of the training subjects using Euclidean distance as the metric for comparison. The training subject with the minimum value of the Euclidean distance is assigned to be the class of the test subject. Thus, for each of the $\mathcal{S}$ subjects, we compute the following:

$$
D_{i}=\sum_{m=1}^{l} \sum_{p=1}^{\mathcal{N}}\left(F_{p k_{m}}^{i}-F_{p k_{m}}^{\prime}\right)^{2} .
$$

The test subject is assigned to the $r^{t h}$ class, if $D_{r}<D_{i}, \forall \mathrm{i}=1,2,3, \ldots, \mathcal{S}$ and $i \neq r$.

\section{Experimental Results}

We next present results from an extensive set of experiments carried out using the proposed method. The algorithm has been implemented in Matlab 7.12.0 (R2010a) on a $2.50 \mathrm{GHz}$ Intel Core i5 processor having 4GB RAM. The experimental setup is described next. 
Two Kinects are positioned at two ends of a narrow pathway, facing each other, each at a height of 2 metres from the ground. The tilt angles of both the Kinects are set to -27 degrees. During both the key pose estimation phase and the gait training phase, only one Kinect camera is employed to record the gait sequence of each subject from the front view as well as the back view. The test set is constructed by allowing each person to walk through the pathway. The data set consists of a total of 45 subjects. Two complete gait cycles $T 1$ and T2 from the front and two complete cycles T3 and T4 from the back, each captured at 30 frames per second (fps), serve as the training data set. The entire video recording procedure for the test cases is repeated for two different walking speeds: normal walk $(N W)$, i.e., at the same speed as that of the training sequence and fast walk $(F W)$, both of which are again recorded under two different frame rate settings: $F 1=30 \mathrm{fps}$ and $F 2=15 \mathrm{fps}$, respectively. For comparative analysis with other recently proposed gait features, we have captured the RGB as well as depth channel information along with skeleton joint coordinates for a total of 17 subjects, selected out of these 45 subjects.

First, we study the performance of the proposed method in case of both normal and fast walk. Results are presented in Table 1 with two different frame rates. The different rows represent various combinations of data sets (T1, T2, T3, T4) used for training as mentioned in the last sub-section. It is observed that quite accurate results are obtained both for normal walk and fast walk when the frame rate during test data capture is kept the same as that during the training data capture. A higher performance is usually achieved with normal walk than with fast walk because fast walk causes greater number of key poses to be missed in a walking sequence. Also, not much degradation is observed by reducing the frame rate to half in the test case. In general, performance of the algorithm improves if the size of the training data is increased.

In most practical situations, we do not need to choose only the subject with the best match. Rather, it is sufficient if the correct class falls within the top $r$ classes predicted by the algorithm. In the present study, we examine how many subjects are correctly classified as the value of $r$ is varied from 1 to 10 . The training set for this experiment consists of $T 1$ and $T 3$ (i.e., one gait cycle each from the front and back views) and the results are shown using the cumulative match characteristic $(C M C)$ curves of Figure1 It is observed that beyond a rank of 5 , almost $98 \%$ accuracy is obtained. Further, similar performance is achieved for both fast walk and slow walk.

Finally, a comparative analysis of performance of the proposed method is made with some of the existing gait recognition approaches, namely, those derived by utilizing the depth data of Kinect like Gait Energy Volume [3] and skeleton co-variance feature [12] along with the widely used Gait Energy Image feature [1]. Results are presented in Table 2. Training set for this experiment consists of $T 1$ and $T 3$. It is seen that although the proposed method takes about 1 second more than each of [1] and [12, it outperforms all of the current state of the art algorithms in terms of accuracy by more than $5 \%$, which is quite significant. 
Table 1. Variation of recognition accuracy (in \%) with frame rate (F1 and F2) for both normal walk $(N W)$ and fast walk $(F W)$ with different training data set (T1, T2, T3 and T4) combinations

\begin{tabular}{c|lc|lc}
\hline \multirow{2}{*}{ Training Data Set } & \multicolumn{2}{|l|}{$F 1$ (frame rate: $30 \mathrm{fps})$} & \multicolumn{2}{|l}{ (frame rate: $15 \mathrm{fps}$ ) } \\
\cline { 2 - 5 } & $N W$ & $F W$ & $N W$ & $F W$ \\
\hline T1 & 80.00 & 77.78 & 82.22 & 66.67 \\
\hline T2 & 75.56 & 84.44 & 84.44 & 62.22 \\
\hline T3 & 80.00 & 84.44 & 80.00 & 68.89 \\
\hline T4 & 80.00 & 73.33 & 68.88 & 71.11 \\
\hline$T 1+T 2$ & 82.22 & 82.22 & 91.11 & 73.33 \\
\hline$T 1+T 3$ & 93.33 & 93.33 & 93.33 & 84.44 \\
\hline$T 2+T 4$ & 93.33 & 91.11 & 91.11 & 77.78 \\
\hline$T 3+T 4$ & 86.67 & 80.00 & 84.44 & 84.44 \\
\hline$T 1+T 2+T 3+T 4$ & 95.56 & 95.56 & 95.56 & 88.89 \\
\hline
\end{tabular}

Table 2. Performance comparison of different gait recognition algorithms

\begin{tabular}{|c|c|c|}
\hline Gait Feature & Accuracy(\%) & Time(secs) \\
\hline Gait Energy Image 1 & 76.47 & 0.45 \\
\hline Gait Energy Volume 3 & 70.59 & 2.33 \\
\hline Skeleton Co-Variance Feature 12 & 76.47 & 0.30 \\
\hline Proposed feature & 82.35 & 1.37 \\
\hline
\end{tabular}

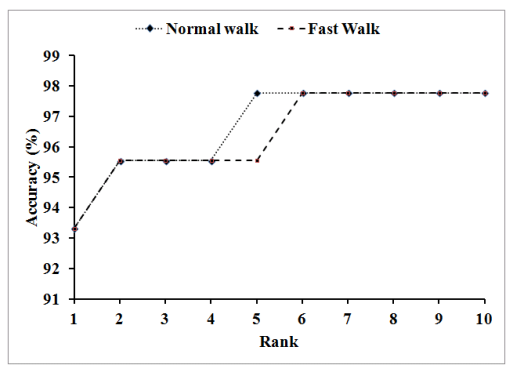

Fig. 1. Cumulative match characteristic curves with two different walking speeds during testing

\section{Conclusion}

In this paper, we have proposed a key pose based approach of gait recognition. The gait features for each key pose are derived by utilizing the skeleton tracking data provided by Kinect. Better skeleton tracking capability of Kinect is expected to improve the performance of the algorithm even further. From the extensive set of experiments, it can be concluded that use of Kinect depth data has an immense potential in frontal gait recognition. The method is also fast enough to achieve meaningful response time. The processing time can be reduced by using coding in $\mathrm{C} / \mathrm{C}++$ instead of Matlab. More experiments, however, need to be conducted with challenging conditions including occlusion, collision, etc. 
Acknowledgement. This work is partially funded by Project Grant No. 22(0554)/11/EMR-II sponsored by the Council of Scientific and Industrial Research, Govt. of India.

\section{References}

1. Han, J., Bhanu, B.: Individual Recognition using Gait Energy Image. IEEE Trans. on Pattern Analysis and Machine Intel. 28(2), 316-322 (2006)

2. Roy, A., et al.: Gait Recognition using Pose Kinematics and Pose Energy Image. Signal Processing 92(3), 780-792 (2012)

3. Sivapalan, S., et al.: Gait Energy Volumes and Frontal Gait Recognition using Depth Images. In: International Joint Conf. on Biometrics, pp. 1-6 (2011)

4. Soriano, M., et al.: Curve Spreads - A Biometric From Front View Gait Video. Pattern Recognition Letters 25(14), 1595-1602 (2004)

5. Barnich, O., et al.: Frontal-View Gait Recognition by Intra- and Inter-Frame Rectangle Size Distribution. Pattern Recognition Letters 30(10), 893-901 (2009)

6. Lee, K., et al.: Frontal View-Based Gait Identification using Largest Lyapunov Exponents. In: Proc. of the Intl. Conf. on Accoustics, Speech, and Signal Processing, pp. $173-176(2006)$

7. Gofferedo, M., et al.: Front-View Gait Recognition. In: Proc. of the IEEE Intl. Conf. on Biometrics: Theory, Applications and Systems, pp. 1-6 (2011)

8. Ryu, J., Kamata, S.: Front View Gait Recognition using Spherical Space Model with Human Point Clouds. In: Eighteenth IEEE International Conf. on Image Processing, pp. 3209-3212 (2011)

9. Zhang, Z.: Microsoft Kinect Sensor and its Effect. IEEE Multimedia 19(2), 4-10 (2012)

10. Chattopadhyay, P., et al.: Pose Depth Volume Extraction from RGB-D Streams for Frontal Gait Recognition. Journal of Visual Communication and Image Representation (2013), doi:10.1016/j.jvcir.2013.02.010

11. Shotton, J., et al.: Real-Time Human Pose Recognition in Parts from Single Depth Images. In: IEEE Conf. on Computer Vision and Pattern Recognition (CVPR), pp. 1297-1304 (2011)

12. Kumar, M.S., Babu, R.V.: Human Gait Recognition using Depth Camera: A Covariance Based Approach. In: Proc. of the Eighth Indian Conference on Computer Vision, Graphics and Image Processing, Art., vol. 20 (2012)

13. Jin, X., Han, J.: K-Means Clustering. In: Encyclopedia of Machine Learning, pp. 563-564. Springer, US (2010) 